\title{
Mécanismes de la persistance de la mémoire immunologique
}

La vaccination est une méthode très efficace de prévention et de diminution de la morbidité et de la mortalité dues à des agents infectieux. Cette efficacité témoigne de l'existence, chez les individus vaccinés, d'un phénomène couramment appelé la mémoire immunologique. En effet, quand un individu rencontre pour la première fois un agent infectieux, la réponse immunologique est peu efficace. Une deuxième rencontre avec le même agent pathogène induit une réponse (à la fois cellulaire et humorale) plus rapide, plus intense et plus spécifique. L'agent pathogène peut ainsi être éradiqué avant l'invasion des tissus de l'hôte, ce qui réduit considérablement ou évite ses effets pathogènes. Il n'est donc pas surprenant que l'étude des mécanismes responsables de l'établissement et du maintien d'une mémoire immunologique efficace constitue un des pôles principaux de la recherche en immunologie. Deux axes principaux sont développés. Le premier consiste à isoler et à caractériser les composants (antigènes) d'un agent infectieux, capables d'induire une réponse protectrice, et à définir les méthodes d'administration les plus efficaces de ces antigènes. Cette démarche consiste, sur le plan fondamental, à étudier l'apprêtement de l'antigène et sa présentation aux cellules immunocompétentes, et peut être décrite, sur un plan plus appliqué, comme "le dessin de vaccins». Le second axe de recherche, tout aussi important, a pour objectif de caractériser les facteurs responsables du maintien dans le temps de la mémoire immunitaire. C'est sur cette partie que nos travaux de recherche se sont focalisés pendant les dernières années.

\section{Problématique}

Quels sont les signaux nécessaires au maintien de la mémoire immunologique? La réponse à cette question a des implications pratiques importantes pour le développement des stratégies vaccinales. Trois possibilités peuvent être envisagées. (1) Il n’y a «mémoire» que si l'antigène persiste dans l'organisme. Cela signifierait que les seules vaccinations totalement efficaces reposent sur l'utilisation de pathogènes atténués, qui pourraient survivre ou se multiplier lentement chez l'individu vacciné, assurant cette charge antigénique persistante. Pour les autres types de vaccins, des rappels vaccinaux fréquents seraient indispensables. L'étude de la persistance antigénique serait donc l'objectif le plus important des recherches. (2) La survie des cellules mémoire n'aurait pas besoin d'antigène spécifique, mais nécessiterait une stimulation antigénique continue, probablement par des antigènes de l'environnement à réactivité croisée avec l'antigène vaccinal. Le maintien de la mémoire dépendrait des signaux induits par le récepteur $\mathrm{T}$, et non plus de la présence de l'antigène, ce qui permettrait une approche vaccinale plus diversifiée. Elle introduirait cependant un caractère plus aléatoire des réponses obtenues car la persistance de la mémoire dépendrait de l'environnement antigénique de l'individu. L'intensité de la réponse primaire (dépendante de l'antigène) ne serait pas nécessairement en relation avec la survie de la mémoire (dépendante de l'environnement), et ces deux paramètres devraient toujours être étudiés séparément. (3) Les cellules «mémoire" peuvent survivre «les yeux fermés» sans qu'aucune sti- mulation, par l'intermédiaire de leur récepteur $\mathrm{T}$, ne soit requise. La stratégie vaccinale reposerait donc sur l'étude du seul paramètre important d'efficacité que serait l'activation du système immunitaire et la production des cellules «mémoire». En effet, la survie des cellules ne poserait aucun problème, puisque toutes les cellules pourraient survivre de la même façon.

\section{Approches expérimentales}

L'identification des mécanismes permettant la pérennité de la mémoire immunologique repose sur l'analyse de deux paramètres: (1) l'évaluation du devenir, dans le temps et l'espace, des lymphocytes $\mathrm{T}$ spécifiques d'un antigène donné après immunisation, ce qui implique de les visualiser; (2) la caractérisation des signaux de l'environnement qui conditionnent la survie des cellules "mémoire».

Des progrès méthodologiques ont permis l'identification des lymphocytes $\mathrm{T}$ spécifiques. On peut d'une part utiliser des souris génétiquement modifiées qui expriment, à la surface de la totalité des lymphocytes $\mathrm{T}$ le même récepteur $\mathrm{T}$ (TcR), spécifique d'un antigène connu. D'autre part, il est possible de suivre, chez une souris normale, les cellules «mémoire» en utilisant la capacité du récepteur $\mathrm{T}$ à reconnaître et à se lier à des molécules du complexe majeur d'histocompatibilité (CMH) présentant l'antigène. Enfin, l'utilisation de tétramères synthétiques des molécules du CMH, liés au peptide antigénique et marqués à la fluorescéine, permet l'identification et le suivi des cellules spécifiques.

L'identification des signaux nécessaires à la survie des cellules $\mathrm{T}$ mémoires a été d'une approche plus 
difficile et a bénéficié récemment de l'obtention, par Béatrice Pérarneau et François Lemonnier à l'Institut Pasteur, de souris dont les molécules du CMH ne sont plus exprimées. En l'absence de ces molécules l'antigène ne peut plus être présenté, et l'étude des signaux permettant la survie des cellules «mémoire» en l'absence d'une stimulation antigénique devient donc possible. L'utilisation de ces souris a permis à deux groupes indépendants, l'un à Paris [1] et l'autre aux États-Unis [2], d'obtenir des résultats semblables avec toutefois quelques différences. Nous avons utilisé des souris transgéniques dont les lymphocytes $\mathrm{T}$ expriment un TcR reconnaissant spécifiquement un peptide de l'antigène mâle, présenté par des molécules du $\mathrm{CMH}$ de classe I H-2D ${ }^{\mathrm{b}}$. Nous avons démontré que les cellules mémoires survivent après leur transfert chez la souris n'exprimant pas $\mathrm{H}_{-}-2 \mathrm{D}^{\mathrm{b}}$, et donc incapable de présenter cet antigène [1]. Les mêmes conclusions ont été obtenues par le groupe de Rafi Ahmed aux États-Unis qui a étudié des cellules mémoires spécifiques des antigènes du virus de la lymphochorioméningite de la souris normale, présentés par le CMH H-2D $\mathrm{D}^{\mathrm{b}}[2]$. Ces cellules, identifiées par le tétramère synthétique CMH-peptide fluorescent, persistent aussi chez la souris $\mathrm{H}_{-}-2 \mathrm{D}^{\mathrm{b}}$ négative, qui n'a aucune possibilité de présenter l'antigène. Ces résultats indiquent que les cellules mémoires survivent sans qu'un contact continu avec l'antigène spécifique soit nécessaire (ce qui exclut la possibilité 1 indiquée plus haut). Le maintien de la mémoire immunologique ne dépend donc pas d'une stimulation antigénique continue, et l'approche vaccinale peut être diverse.

Il restait donc à déterminer si la survie des cellules «mémoire» requiert ou non des signaux induits par les TcR. Ces cellules ont donc été transférées chez des souris $D^{b-} K^{b-} \beta_{2} m$ dépourvues de toutes les molécules du CMH de classe I et dont les TcR ne peuvent plus être activés [1]. Nos résultats ont montré que ces signaux étaient nécessaires puisque nos cellules ne survivent pas après leur transfert chez la souris $D^{b-} K^{b-} \beta_{2} m^{-}$. Des résultats identiques, obtenus avec le même clone de cellules $\mathrm{T}$, ont été publiés [3]. Le groupe de Rafi Ahmed émettait en revanche des conclusions opposées puisque sa population de cellules "mémoire» survivait à long terme après transfert chez une souris dépourvue de molécules du CMH I [2].

Une des hypothèses permettant d'expliquer ces différences serait le type de population étudiée: un seul clone dans notre cas, et une population polyclonale hétérogène dans l'autre. On observe en effet dans les premiers jours suivant la transplantation de cette population polyclonale, une disparition de $50 \%$ à $90 \%$ des cellules transférées, suivie d'une expansion des cellules résiduelles. Ces résultats pourraient indiquer que les cellules «mémoire»sont hétérogènes: certaines ne survivraient qu'en présence d'interactions TcRCMH classe I, les autres pouvant s'en passer. La fraction des cellules CD8 ${ }^{+}$ qui persiste pourrait soit reconnaître le CMH de classe II, encore présent chez la souris $D^{b-} K^{b-} \beta_{2} m^{-}$, soit être vraiment indépendante de l'interaction TcR-CMH.

Les différences entre nos résultats expérimentaux pourraient, hélas, avoir des explications plus triviales, liées à des problèmes méthodologiques. Les souris receveuses, dépourvues de molécules CMH de classe I, doivent en effet être irradiées pour empêcher leurs cellules $\mathrm{T}$ de rejeter les cellules mémoires transférées qui, elles, expriment le $\mathrm{CMH}$ de classe I. L'irradiation peut induire deux types d'artefacts: (1) la destruction de la paroi intestinale, et l'invasion de l'hôte par des bactéries de la flore intestinale qui en résulte, peuvent induire une prolifération importante des cellules "mémoire» transplantées. Nous ignorons si, dans l'étude américaine, un traitement antibiotique a été utilisé pour éviter une telle réaction. (2) L'irradiation induit une contraction importante des organes lymphoïdes qui sont vidés de leurs cellules. Cette contraction, associée à la multiplication des cellules transférées, rendrait probable le fait que ces dernières (qui expriment le CMH de classe I) aient l'opportunité d'interagir avec les cellules issues des ganglions.

Ces limitations expliquent pourquoi les mécanismes contrôlant la persistance de la réponse mémoire ne sont pas encore établis. Leur identification nécessitera vraisemblablement l'étude de la survie d'une population mémoire polyclonale dans des hôtes déficients en molécules CMH de classe I et de classe II, et ce, en l'absence d'irradiation.

1. Tanchot C, Lemonnier FA, Pérarneau B, Freitas AA, Rocha B. Differential requirements for survival and proliferation of CD8 naive or memory T cells. Science 1997; 276: 2057-62.

2. Murali-Krishna K, Lau LL, Sambhara S, Lemonnier F, Altman J, Ahmed R. Persistence of memory CD8 T cells in MHC class I-deficient mice. Science 1999; 286: 1377-81

3. Markiewicz MA, Girao C, Opferman JT, et al. Long-term $\mathrm{T}$ cell memory requires the surface expression of self-peptide/major histocompatibility complex molecules. Proc Natl Acad Sci USA 1998; 95 : 3065-70.

\section{Benedita Rocha}

Inserm U. 345, Institut Necker, 156, rue de Vaugirard, 75730 Paris Cedex 15, France.

\section{LE CYCLE CELLULAIRE, Institut Curie, Paris $1^{\text {er }}$ et 2 février 2001}

\section{Société Française de Génétique}

Société de Biologie Cellulaire de France

Contact: Michel Werner ou Carl Mann, SBGM, Bâtiment 142, CEA/Saclay, f-91191 Gif-sur-Yvette Cedex, France. Télécopie: + 33 (0) 169084712 - E-mail: CC2001@matthieusaclay.cea.fr - WEB: http://persolibertysurf.fr/sfg/colloq2001.html 